Classification

Physics Abstracts

71.55

\title{
Analyse d'une méthode D.L.T.S. utilisant une détection synchrone mesurant l'harmonique deux du signal de capacité
}

\author{
A. Le Bloa et P. N. Favennec $\left({ }^{*}\right)$ \\ Groupe d'Electronique et de Physique des Matériaux, Université de Rennes I, \\ Avenue du Général Leclerc, 35042 Rennes Cedex, France \\ (*) Groupement I.C.M./T.O.H., Centre National d'Etudes des Télécommunications, \\ Lannion B, 22301 Lannion, France
}

(Reçu le 15 décembre 1981, révisé le 21 mai 1982, accepté le 21 juillet 1982)

\begin{abstract}
Résumé. - Nous décrivons une méthode D.L.T.S. utilisant une détection synchrone dont la mise en œuvre est simple; cette méthode réduit l'influence sur les mesures de la réponse transitoire du capacimètre et élimine la composante continue de la capacité. La détection de l'harmonique deux présente alors une meilleure sélectivité que la détection du fondamental. La limitation à une demi-période du signal de capacité à l'entrée de la détection synchrone réduit le bruit de fond pour les coefficients de Fourier $a_{1}, a_{2}, b_{2}$ de ce signal. Une seule remontée de température est suffisante pour déterminer la signature des défauts. Nous caractérisons trois défauts d'énergie d'activation respective : 0,$22 ; 0,29 ; 0,39 \mathrm{eV}$ dans un échantillon de $\mathrm{Ga}$ As multi-implanté en oxygène.
\end{abstract}

\begin{abstract}
A D.L.T.S. method using a lock-in amplifier is described whose setting up is simple. This method reduces the influence of the capacimeter transient response upon measurements and suppresses the direct component of the capacitance signal. The detection of second harmonic permits a better selectivity than the detection of the fundamental. Limiting to half a period the capacitance signal steps down the noise in measuring the Fourier coefficients $a_{1}, a_{2}, b_{2}$ of this signal. A single temperature scanning is sufficient for determining the defect " signature ". By way of example three defects are characterized in an oxygen multi-implanted Ga As sample : respective activation energy of them are $0.22,0.29$ and $0.39 \mathrm{eV}$.
\end{abstract}

1. Introduction. - La spectroscopie des niveaux profonds (Deep Level Transient Spectroscopy ou D.L.T.S.) imaginée par Lang [1] permet de caractériser les défauts d'un matériau semiconducteur par les valeurs, en fonction de la température, de la probabilité d'émission d'un porteur et par l'énergie d'activation de cette probabilité. Ces caractéristiques sont déterminées, en fonction de la température, à partir de la réponse en capacité d'une diode à un signal impulsionnel périodique de polarisation, le substrat de la diode étant le semiconducteur étudié. Au cours d'une période $\left(T_{0}\right)$ une phase de capture de porteurs par les pièges est suivie de l'émission de ces porteurs vers leur bande, produisant des variations transitoires de la capacité de la diode.

Plusieurs méthodes ont été proposées pour traiter ce signal, Miller et al. [2] ont présenté une méthode de corrélation qu'ils ont comparée à la méthode de Lang et à celle de Kimmerling qui met en œuvre une détection synchrone sélective; cette dernière méthode a, de son côté, été discutée par Schott et al. [3] et améliorée par Day et al. [4] en diminuant notablement l'influence de la réponse transitoire du capacimètre sur les mesures. Pons [5] a aussi décrit une méthode utilisant une détection synchrone à large bande où le signal de capacité produit pendant le remplissage des défauts par des électrons ou des trous et la réponse transitoire du capacimètre ne donnent aucune contribution au signal D.L.T.S.

Jusqu'à présent seule la variation, en fonction de la température, soit de l'amplitude $A_{1}$ du fondamental du signal de capacité (détection synchrone sélective), soit des signaux en phase et en quadrature avec le signal de capacité (détection synchrone à large bande) ont été utilisés pour déterminer les probabilités d'émissions des porteurs. Nous montrons comment une détection synchrone, mesurant $A_{2}$ l'amplitude, $\phi_{2}$ la phase de l'harmonique deux du signal de capacité (ou $A_{2} \cos \phi_{2}$ et $A_{2} \sin \phi_{2}$ ) peut être utilisée pour réaliser une méthode D.L.T.S. de mise en ouvre 
simple; nous analyserons ensuite les différents types de spectres qui peuvent être obtenus, avant d'exposer quelques résultats expérimentaux.

2. Méthode de mesure. - Nous précisons ici le principe de la méthode de mesure, les principales caractéristiques du montage utilisé et les valeurs des signaux de sortie de la détection synchrone, avant de montrer que l'utilisation de l'harmonique deux permet des mesures plus précises des grandeurs à déterminer que la détection de la fondamentale.

2.1 Principe De LA MESURE. - La diode est initialement polarisée en inverse et sa capacité est alors $C_{0}$. L'impulsion de tension destinée à remplir les pièges diminue cette polarisation inverse ou même polarise la diode en direct ce qui modifie sa capacité. Après la fin de l'impulsion, la capacité revient à sa valeur initiale en suivant une loi qui, pour un type donné de défauts, s'exprime par :

$$
\Delta C(t)=\Delta C_{0}[1-\exp (-t / \tau)]
$$$$
\text { avec } \quad \tau=\left(e_{\mathrm{n}}+e_{\mathrm{p}}\right)^{-1}
$$

où $t$ est le temps, $\Delta C_{0}$ l'amplitude de la variation de la capacité sous l'effet de l'impulsion de polarisation, $e_{\mathrm{n}}$ et $e_{\mathrm{p}}$ les probabilités d'émissions respectives d'un électron et d'un trou.

Si $\Delta C_{0}$ est indépendant de la température $T$, on peut déduire de l'expression (1) la valeur de $\tau$ à chaque température, soit $e_{\mathrm{n}}$ si $e_{\mathrm{n}} \gg e_{\mathrm{p}}$, soit $e_{\mathrm{p}}$ si $e_{\mathrm{p}} \gg e_{\mathrm{n}}$; on peut alors évaluer l'énergie d'activation $E_{\mathrm{a}}$ de $e_{\mathrm{n}}$ ou $e_{\mathrm{p}}$ si l'on admet [6] :

$$
e_{\mathrm{n}}=\gamma \sigma_{\infty} T^{2} \exp \left(-E_{\mathrm{a}} / k T\right)
$$

avec

$\gamma:$ constante dépendant du semiconducteur

$k$ : constante de Boltzmann

$\sigma_{\infty}$ : section efficace de capture apparente indépendante de la température.

2.2 Montage. - Le schéma synoptique du montage et le diagramme temporel des signaux sont

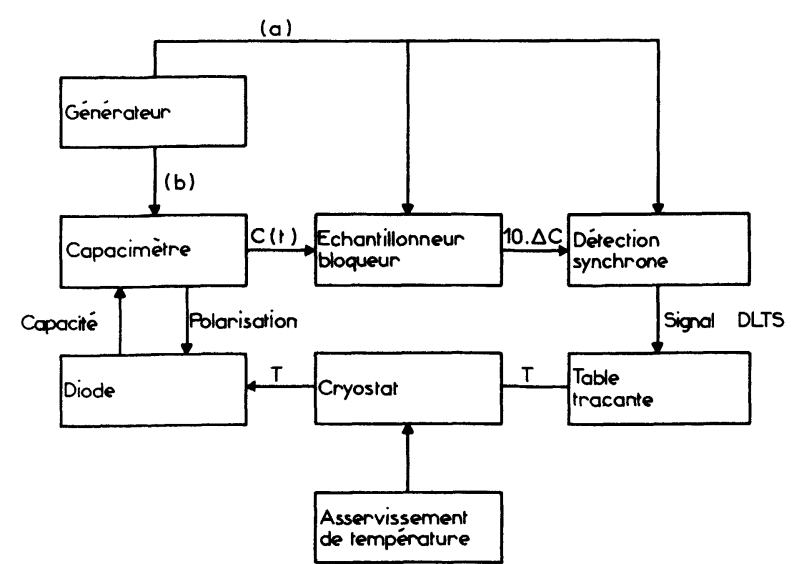

Fig. 1. - Schéma synoptique de l'appareil.

[Block diagram of the system.] présentés respectivement aux figures 1 et 2 . Un générateur d'impulsion fournit :

- une tension carrée $(a)$ (Figs. 1 et $2 a$ ) servant de signal de référence à la détection synchrone et commandant l'échantillonneur-bloqueur;

- une impulsion (b) (Fig. 2b), de largeur, d'amplitude et de retard réglables, de même période que $(a)$, polarisant la diode.

L'échantillonneur-bloqueur ne relie la sortie du capacimètre à l'entrée de la détection synchrone que pendant la deuxième demi-période; dans la première l'échantillonneur-bloqueur conserve à l'entrée de la détection synchrone la valeur de la capacité à l'instant $T_{0}$ (Fig. $2 d$ ); la valeur moyenne du signal devient ainsi voisine de zéro.

Un délai $t_{\mathrm{d}}$ (Fig. $2 b$ ) sépare la fin de l'impulsion de polarisation de l'instant où l'échantillonneur-bloqueur établit la liaison entre la sortie du capacimètre et l'entrée de la détection synchrone. Le but de ce délai est de rendre négligeable l'influence de la réponse transitoire du capacimètre sur les mesures. Comme Day et al. [4] nous choisissons $t_{\mathrm{d}}=1,6 \mathrm{~ms}$ (capacimètre Boonton $72 \mathrm{BD}$ ); nous donnons en appendice quelques indications supplémentaires sur ce choix.

En réalité nous avons monté deux échantillonneursbloqueurs en cascade pour améliorer la précision des mesures aux basses fréquences (diminution de la perte de tension pendant le blocage et diminution des erreurs d'offset au passage de l'échantillonnage au blocage) ; le gain du premier échantillonneur-bloqueur est de dix et celui du second de un, la durée d'échantillonnage de $7 \mu$ s environ.

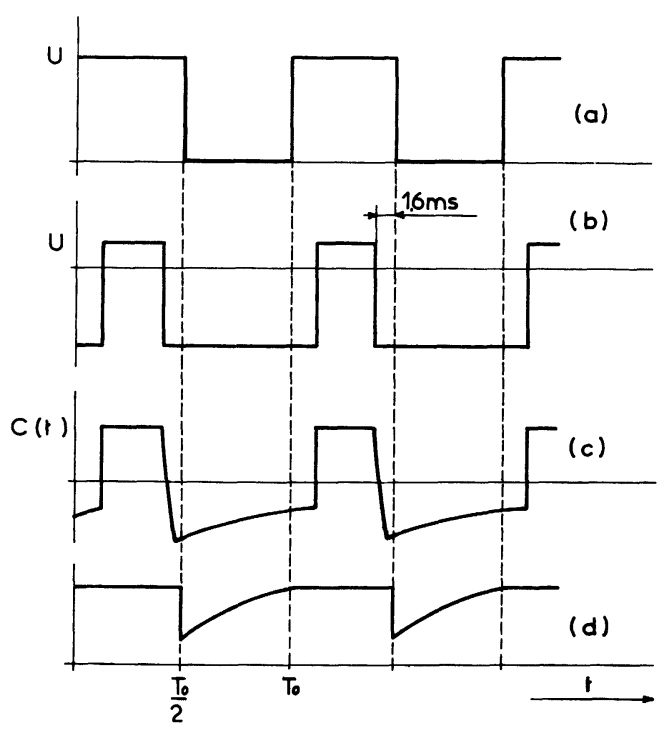

Fig. 2. - Chronogramme des signaux : (a) Signal de commande de l'échantillonneur-bloqueur et signal de référence pour la détection synchrone; $(b)$ polarisation de la diode; (c) signal de sortie du capacimètre; (d) signal de sortie de l'échantillonneur-bloqueur.

[Signal chronogram : (a) Control signal of the sample-hold module and reference signal of the lock-in amplifier; $(b)$ diode bias ; $(c)$ output signal of the capacimeter ; $(d)$ output signal of the sample-hold module.] 
Le parti que nous avons pris de n'admettre le signal de capacité à l'entrée de la détection synchrone que pendant une demi-période simplifie la mise en ouvre de la méthode; en effet les impulsions de tension de polarisation $(b)$ et de commande de l'échantillonneurbloqueur (a) sont directement fournis par le générateur; d'autre part l'expression des signaux de sorties, notamment lorsqu'on détecte l'harmonique deux, est particulièrement simple.

2.3 EXPRESSION DES SIGNAUX DE SORTIE DE LA DÉTECTION SYNCHRONE. - Le signal de sortie de la détection synchrone est la superposition des réponses qu'elle donne des diverses variations exponentielles de la capacité produites par les divers types de défauts. Selon la relation (1) le signal d'entrée de la détection synchrone pour un seul type de défauts (Fig. $2 d$ ) est :

$$
f(t)=\mid \begin{array}{r}
\Delta C_{0}\left\{1-\exp \left[-\left(t_{\mathrm{d}}+\frac{T_{0}}{2}\right) / \tau\right]\right\} \\
\text { pour } 0<t<\frac{T_{0}}{2} \\
\Delta C_{0}\left\{1-\exp \left[-\left(t+t_{\mathrm{d}}-\frac{T_{0}}{2}\right) / \tau\right]\right\} \\
\text { pour } \frac{T_{0}}{2}<t<T_{0} .
\end{array}
$$

Selon le modèle de détection synchrone diverses sorties sont disponibles. Avec l'appareil que nous avons utilisé (ITHACO 393) nous pouvons choisir de faire apparaître aux sorties $A \cos \phi$ ou $A \sin \phi$ la valeur des premiers coefficients soit de la fondamentale $\left(a_{1}\right.$ et $\left.b_{1}\right)$ soit de l'harmonique deux $\left(a_{2}, b_{2}\right)$ du développement en série de Fourier de $f(t)$.

$$
f(t)=\frac{a_{0}}{2}+\sum_{n=1}^{\infty} a_{n} \cos (n \omega t)+b_{n} \sin (n \omega t)
$$

avec

$$
\omega=2 \pi / T_{0}
$$

et

$$
\begin{aligned}
& a_{n}=\frac{2}{T_{0}} \int_{0}^{T_{0}} f(t) \cos (n \omega t) \mathrm{d} t \\
& b_{n}=\frac{2}{T_{0}} \int_{0}^{T_{0}} f(t) \sin (n \omega t) \mathrm{d} t
\end{aligned}
$$

Les expressions de ces coefficients pour $f(t)$ définie en (3) ainsi que celles de l'amplitude $A_{2}=\sqrt{a_{2}^{2}+b_{2}^{2}}$ et de la phase $\phi_{2}\left(\operatorname{tg} \phi_{2}=a_{2} / b_{2}\right)$ sont présentées dans le tableau I, en fonction de la constante de temps normalisée $x=\omega \tau$ et pour un signal de référence proportionnel à $\sin \omega t$; dans le cas d'une référence proportionnelle à $\cos \omega t$, les sorties où sont disponibles les $a_{i}$ et $b_{i}$ seraient inversées.

A l'exception de $\phi_{2}$ chaque expression du tableau I est représentée en fonction de la température par une courbe en forme de pic $(x=\omega \tau$ est une fonction de $T)$.

Le terme correctif introduit par l'intervalle de temps $t_{\mathrm{d}}, \exp \left(-\omega t_{\mathrm{d}} / x\right)$, est le même pour tous les signaux, exception faite de $\phi_{2}$ qui en est indépendant ; $\phi_{2}$ est en outre indépendant de $\Delta C_{0}$. Si dans certaines conditions expérimentales $\Delta C_{0}$ varie avec la température, la mesure de $\phi_{2}$ permet une détermination plus précise de $e_{\mathrm{n}}$ et de $E_{\mathrm{a}}$ que les autres coefficients. La mesure de $\phi_{2}$ n'est pas précise lorsque $a_{2}$ et $b_{2}$ ont un faible niveau, le diviseur de la détection synchrone ne fonctionnant pas alors dans de bonnes

Tableau I. - Expression des signaux disponibles aux sorties d'une détection synchrone pour l'entrée $f(t)$ (expression (3)); le nom des sorties indiquées se rapporte à l'appareil ITHACO 393.

[Expression of the available output signals of a lock-in amplifier for the input $f(t)$ (expression (3)); the name of the indicated outputs refers to the ITHACO 393 instrument.]

Coefficients

Nom des sorties

$$
\begin{array}{cc}
a_{1} & \frac{\Delta C_{0}}{\pi} \exp \left(-\omega t_{\mathrm{d}} / x\right)\left[\frac{x(1+\exp (-\pi / x))}{1+x^{2}}\right] \\
a_{2} & -\frac{\Delta C_{0}}{\pi} \exp \left(-\omega t_{\mathrm{d}} / x\right)\left[\frac{x(1-\exp (-\pi / x))}{1+4 x^{2}}\right] \\
b_{1} & \frac{\Delta C_{0}}{\pi} \exp \left(-\omega t_{\mathrm{d}} / x\right)\left[-2 \exp (-\pi / x)+\frac{x^{2}(1+\exp (-\pi / x))}{1+x^{2}}\right] \\
b_{2} & -\frac{\Delta C_{0}}{\pi} \exp \left(-\omega t_{\mathrm{d}} / x\right)\left[\frac{2 x^{2}(1-\exp (-\pi / x))}{1+4 x^{2}}\right] \\
A_{2} & \frac{\Delta C_{0}}{\pi} \exp \left(\omega t_{\mathrm{d}} / x\right)\left[\frac{x(1-\exp (-\pi / x))}{\left(1+4 x^{2}\right)^{1 / 2}}\right] \\
\phi_{2} & \operatorname{arctg}(1 / 2 x) \quad\left(b_{2} \neq 0\right)
\end{array}
$$


conditions. Toutes ces remarques sont valables pour la phase $\phi_{1}$ du fondamental, mais comme nous le verrons par la suite la mesure de celle-ci est perturbée par un bruit de fond plus important que la mesure de $\phi_{2}$; l'expression de $\phi_{1}$ est aussi plus complexe que celle de $\phi_{2}$.

Le terme correctif $\exp \left(-\omega t_{\mathrm{d}} / \tau\right)$ est identique à celui introduit par Day et al. [4] mais la fonction

$$
\begin{gathered}
g(t)=\exp \left(-t_{\mathrm{d}} / \tau\right) \exp \left[-\left(t+\frac{T_{0}}{2}\right) / \tau\right] \\
\left(-\frac{T_{0}}{2} \leqslant t<\frac{T_{0}}{2}\right)
\end{gathered}
$$

qu'ils choisissent comme expression du signal d'entrée de la détection synchrone n'est pas tout à fait adéquate : en effet, pendant l'intervalle de temps $t_{\mathrm{d}}$ où la porte reliant la sortie du capacimètre à l'entrée de la détection synchrone est fermée, le signal d'entrée de la détection synchrone ne peut être exprimé par $g(t)$.

Dans notre méthode le signal échantillonné à l'instant $T_{0}$ est conservé à l'entrée de la détection synchrone par l'échantillonneur-bloqueur pendant une demi-période $\left(\left[0, T_{0} / 2[)\right.\right.$ (Fig. $\left.2 d\right)$; ce signal est donc une impulsion de tension de la durée d'une demipériode dont l'amplitude varie aléatoirement à chaque période, particulièrement lorsque le signal de sortie du capacimètre est noyé dans le bruit, il en résulte à la sortie de la détection synchrone un bruit qui se superpose au bruit de l'appareillage ; ce bruit d'échantillonnage du signal a été discuté par Crowell et al. [7]. Comme le signal échantillonné (intervalle $\left[0, T_{0} / 2[\right.$ ) est carré il ne contribue qu'au coefficient de Fourier $b_{1}$ : autrement dit les valeurs de $a_{1}, a_{2}, b_{2}$ proviennent de la restriction de $f(t)$ (Fig. $2 d)$ à l'intervalle de temps $\left[T_{0} / 2, T_{0}[\right.$. Il est donc particulièrement intéressant de mesurer $a_{1}, a_{2}, b_{2}$ puisque le bruit est moindre que pour $b_{1}$.

Pour comparer la résolution de notre méthode à la résolution des méthodes proposées par Crowell et

Tableau II. - Figure de mérite $F$ et variations asymptotiques des coefficients aux valeurs élevées et faibles de la constante de temps $\tau$; l'ordre du filtre dans la nomenclature de Crowell et al. [7] est indiqué.

[Figure of merit $F$ and asymptotic variations of the coefficients with high and low values of the time constant $\tau$; the filter order in the Crowell et al. [7] nomenclature is indicated.]

\begin{tabular}{ccccc} 
Coefficients & $F$ & $\begin{array}{c}\text { Valeurs } \\
\text { élevées } \\
\text { de } \tau\end{array}$ & $\begin{array}{c}\text { Valeurs } \\
\text { faibles } \\
\text { de } \tau\end{array}$ & $\begin{array}{c}\text { Ordre } \\
\text { du } \\
\text { filtre }\end{array}$ \\
\hline$a_{1}$ & 0,119 & - & - & - \\
$a_{2}$ & 0,057 & $\tau^{-1}$ & $\tau$ & 1 \\
$b_{2}$ & 0,090 & $\tau^{-1}$ & $\tau$ & 2 \\
$A_{2}$ & 0,0683 & $\tau^{-1}$ & $\tau$ & 1
\end{tabular}

al. [7], nous donnons dans le tableau II (pour $t_{\mathrm{d}}=0$ ) les valeurs de la figure de mérite $F$ définie par ces auteurs pour les coefficients précédents où seul $a_{1}$ a une fonction de pondération (cosinusoïdale) considérée par eux ; la figure de mérite que nous calculons pour $a_{1}$ est un peu supérieure à celle qu'ils ont trouvée $(0,112)$.

Nous présentons également dans le tableau II, les variations asymptotiques des coefficients pour les valeurs élevées et faibles de $\tau$. Nous y ajoutons l'ordre du filtre défini par la nomenclature de Crowell et al. [7], en prenant $t_{\mathrm{d}}=0$; la détection synchrone est un filtre du premier ordre lorsqu'elle mesure $a_{1}, A_{2}$, $b_{2}$; mais les variations de ce dernier coefficient, aux petites valeurs de $\tau$ sont en $\tau^{2}$ au lieu de $\tau$ : la sélectivité est alors meilleure pour $b_{2}$ que pour un filtre du premier ordre. La nomenclature des systèmes filtrants basée sur leur réponse aux valeurs de $\tau$ élevées est donc insuffisante pour caractériser leur sélectivité ; la détection synchrone mesurant $a_{2}$ est un filtre du $2^{\mathrm{e}}$ ordre : la sélectivité est donc meilleure pour $a_{2}$ et aussi pour $b_{2}$ (harmonique 2), que pour les autres coefficients, mais leur figure de mérite est également plus petite.

Dans la méthode de Pons [5], le signal D.L.T.S. est la différence entre les signaux de sortie d'une détection synchrone double phase : il retranche la composante en quadrature $(B)$ de la composante en phase avec le signal de référence; cette procédure n'est valable que pour une détection synchrone à large bande dont le signal de sortie est la somme des réponses qu'elle donne de la fondamentale et de tous les harmoniques du signal d'entrée; mais en général, une détection synchrone est sélective et sa réserve dynamique est nettement meilleure que celle d'une détection synchrone à large bande : le signal de sortie est alors proportionnel, suivant le réglage, soit à la fondamentale, soit à l'harmonique deux du signal d'entrée. Lorsqu'on détecte la fondamentale, la différence $A-B$ déduite des résultats de Day et al. [4] présente un minimum négatif $\left(B>A\right.$ lorsque $\left.x=\omega / e_{\mathrm{n}}<1\right)$ et un maximum positif ; cela peut entraîner des erreurs dans l'interprétation du spectre D.L.T.S. Il faut noter également qu'une détection synchrone sélective donne de l'impulsion de capacité produite pendant le remplissage des défauts par des électrons ou des trous (Fig. 2), des réponses $A$ et $B$ telles que $A-B \neq 0$; en effet, la différence entre les fonctions de pondération de $A$ (sinusoïde) et $B$ (cosinusoïde) n'est plus nulle pendant cette impulsion de capacité comme pour une détection synchrone à large bande; ce signal parasite peut entraîner des erreurs sur la détermination de l'énergie d'activation $E_{\mathrm{a}}$ lorsque la durée de l'impulsion de capacité précédente n'est pas négligeable devant la période $T_{0}$ (Fig. 2); il est donc intéressant d'utiliser un échantillonneur-bloqueur entre le capacimètre et la détection synchrone sélective pour éliminer ce signal parasite et choisir la sensibilité de la détection synchrone convenant le mieux au signal exponentiel de capacité. 


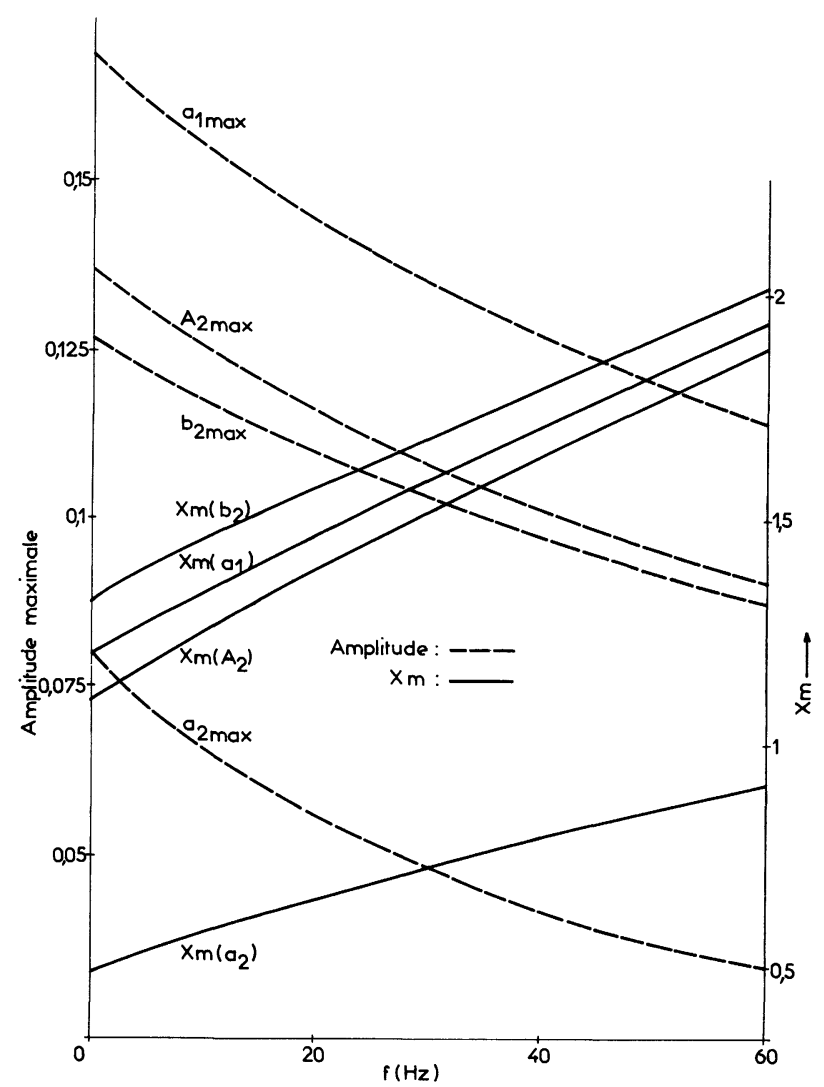

Fig. 3. - Variations en fonction de la fréquence de :l'amplitude maximale (pour $\Delta C_{0}=1$ ) de $a_{1}, a_{2}, b_{2}, A_{2}$; - $x_{\mathrm{m}}$, valeur de $x=\omega \tau$ correspondant à ces maximums.

[Variation versus frequency of : - maximum amplitude (for $\Delta C_{0}=1$ ) of $a_{1}, a_{2}, b_{2}, A_{2} ;-x_{\mathrm{m}}$, value of $x=\omega \tau$ corresponding to these maximums.]

2.4 PARTICULARITÉS DES EXPRESSIONS DES COEFFICIENTS DE FOURIER. - Nous présentons figure 3 les variations, en fonction de la fréquence, du maximum de $a_{1}, a_{2}, b_{2}, A_{2}$ (ces maximums sont nécessaires pour évaluer $e_{\mathrm{n}}$ ) et des valeurs de $x_{\mathrm{m}}$ à ce maximum; la diminution de ces valeurs maximales lorsque la fréquence augmente implique celle du facteur de mérite, compensée par l'accroissement de la sélectivité : en effet, le rapport $t_{\mathrm{d}} / T_{0}$ devenant plus grand, lorsque la fréquence augmente, rend plus abrupt le pic aux valeurs faibles de $\tau$ [7].

Nous présentons également (Fig. 4) pour GaAs, à la fréquence de $20 \mathrm{~Hz}$, les valeurs du couple $\left(E_{\mathrm{a}}, \sigma_{\infty}\right)$ correspondant à une valeur donnée, soit de la température $T_{\mathrm{m}}$ du maximum de $a_{2}\left(a_{2, \mathrm{~m}}\right)$, soit de la largeur en température à mi-hauteur $\Delta T=T_{+}-T_{-}$de ce coefficient, $T_{+}$et $T_{-}\left(T_{+}>T_{-}\right)$sont les températures où la valeur de $a_{2}$ vaut $a_{2, \mathrm{~m}} / 2$. Dans la représentation semi-logarithmique utilisée, les courbes de la famille $T_{\mathrm{m}}=$ Cte sont des droites; en effet, on déduit de $x=\omega \tau$ et de l'équation (2) que :

$$
\log \sigma_{\infty}=\log \left[\omega /\left(\gamma x_{\mathrm{m}} T_{\mathrm{m}}^{2}\right)\right]+E_{\mathrm{a}} /\left(2,3 k T_{\mathrm{m}}\right) .
$$

On notera que si deux pics du spectre ont leur maximum à la même température $T_{\mathrm{m}}$, le moins large cor-

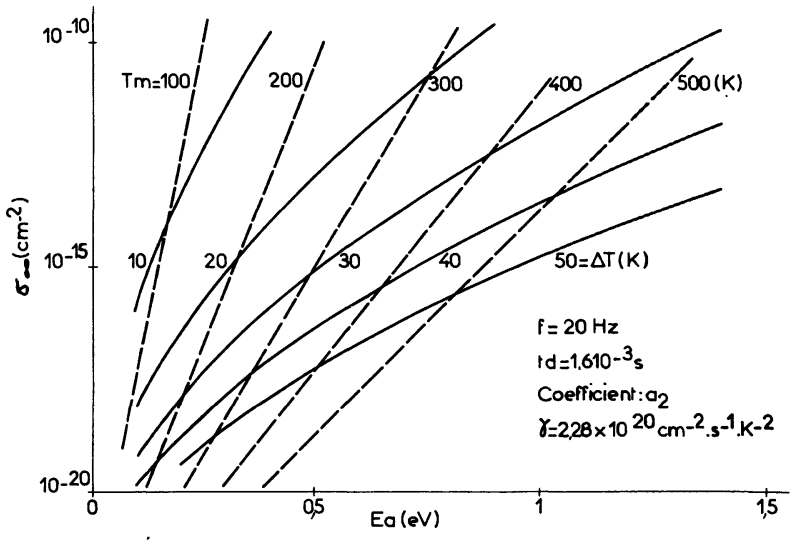

Fig. 4. - Couples $\left(E_{\mathrm{a}}, \sigma_{\infty}\right)$ associés à une valeur donnée soit de la température $T_{\mathrm{m}}$ du maximum de $a_{2}$, soit de $\Delta T$ largeur à mi-hauteur de $a_{2}$ pour une fréquence de $20 \mathrm{~Hz}$ et $\gamma=2,28 \times 10^{20} \mathrm{~cm}^{-2} \cdot \mathrm{s}^{-1} \cdot \mathrm{K}^{-1}$ pour GaAs [6].

[Pairs $\left(E_{\mathrm{a}}, \sigma_{\infty}\right)$ associated with a given value of either $T_{\mathrm{m}}$, temperature of the maximum of $a_{2}$, or $\Delta T$, half-height width of $a_{2}$, in the case of frequency $f=20 \mathrm{~Hz}$ and $\gamma=2.28 \times 10^{20} \mathrm{~cm}^{-2} \cdot \mathrm{s}^{-1} \cdot \mathrm{K}^{-1}$ for GaAs [6].]

respond à la plus grande énergie d'activation et à la plus grande valeur de $\sigma_{\infty}$.

L'influence de la fréquence, à $\sigma_{\infty}$ donnée, sur les mesures est représentée (Fig. 5) par les variations, en fonction de $E_{\mathrm{a}}$, de $T_{\mathrm{m}}$ aux fréquences de 1 et $60 \mathrm{~Hz}$ et de $\Delta T$ à la fréquence de $1 \mathrm{~Hz}$ seulement, car les variations de $\Delta T$ avec la fréquence sont faibles; en effet en désignant par $x_{+}$et $x_{-}$les valeurs de $x$ correspondant à $T_{+}$et $T_{-}$, nous déduisons de $x=\omega / e_{\mathrm{n}}$ et de l'équation (2) que :

$$
\operatorname{Ln}\left(\frac{x_{-}}{x_{+}}\right)=2 \operatorname{Ln}\left(\frac{T^{+}}{T^{-}}\right)+\frac{E_{\mathrm{a}}}{k}\left(\frac{1}{T_{-}}-\frac{1}{T_{+}}\right) .
$$

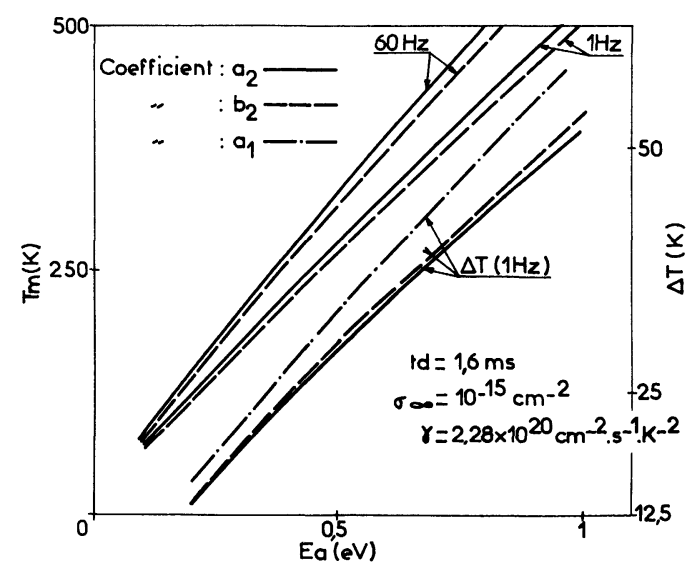

Fig. 5. - Variations, en fonction de l'énergie d'activation $E_{\mathrm{a}}$ de : $-T_{\mathrm{m}}$ température du maximum de $a_{2}(-)$ et $b_{2}(---) ;-\Delta T$ largeur à mi-hauteur de $a_{2}$ (-), $b_{2}(---)$ et $a_{1}(-\cdot-)$.

[Variation versus activation energy $E_{\mathrm{a}}$ of : $-T_{\mathrm{m}} \operatorname{maxi}$ mum temperature of $a_{2}(-)$ and $b_{2}(---) ;-\Delta T$ half-height width of $a_{2}(-), b_{2}(----)$ and $a_{2}(-\cdot-)$.] 
Dans cette relation, les faibles variations de $\operatorname{Ln}\left(x_{-} / x_{+}\right)$ (pour $a_{2}$ : de 2,42 à 1,87 pour $f$ variant de 1 à $60 \mathrm{~Hz}$ ) et la grande valeur de $\left(E_{\mathrm{a}} / k\right)\left(E_{\mathrm{a}} / k>10^{3}\right.$ pour $\left.E_{\mathrm{a}}>0,1 \mathrm{eV}\right)$ impliquent une faible variation de $\Delta T$ avec la fréquence. Pour chaque coefficient $a_{2}$ ou $b_{2}$, l'écart entre les deux courbes $T_{\mathrm{m}}=f\left(E_{\mathrm{a}}\right)$ correspondant aux fréquences de 1 et $60 \mathrm{~Hz}$ croît avec $E_{\mathrm{a}}$ (Fig. 5), c'est-à-dire que le déplacement d'un pic en fonction de la fréquence est d'autant plus grand que $E_{\mathrm{a}}$ est plus élevée ; ainsi deux pics, superposés dans un spectre D.L.T.S. et correspondant à deux défauts d'énergie d'activation voisine $E_{\mathrm{a}_{1}}$ et $E_{\mathrm{a}_{2}}\left(E_{\mathrm{a}_{2}}>E_{\mathrm{a}_{1}}\right)$ et de même section efficace $\sigma_{\infty}$, seront mieux séparés si la mesure est pratiquée à $60 \mathrm{~Hz}$ : le déplacement vers les hautes températures du pic correspondant au défaut d'énergie d'activation $E_{\mathrm{a}_{2}}$ est plus important que celui du défaut d'énergie d'activation $E_{\mathrm{a}_{1}}$. Lorsque les défauts ont des sections efficaces $\sigma_{\infty}$ différentes, le déplacement relatif en fonction de la fréquence est plus complexe, comme nous l'avons montré ailleurs [8].

Pour un défaut donné, l'augmentation de $T_{\mathrm{m}}$ en fonction de la fréquence de mesure est légèrement plus importante pour $a_{2}$ que pour $b_{2}$ et $a_{1}$ (Fig. 5); en revanche $\Delta T\left(E_{\mathrm{a}}, \sigma_{\infty}\right.$ données $)$ est plus faible mesurée à partir de $a_{2}$ que mesurée à partir de $b_{2}$ et surtout de $a_{1}$ (Fig. 5); on en déduit que le spectre de $a_{2}$ présente une meilleure séparation de pics voisins que celui de $b_{2}$ : ceci est illustré par la figure 6 ; en revanche $b_{2}$ permet une meilleure séparation des pics que $a_{1}$ : en

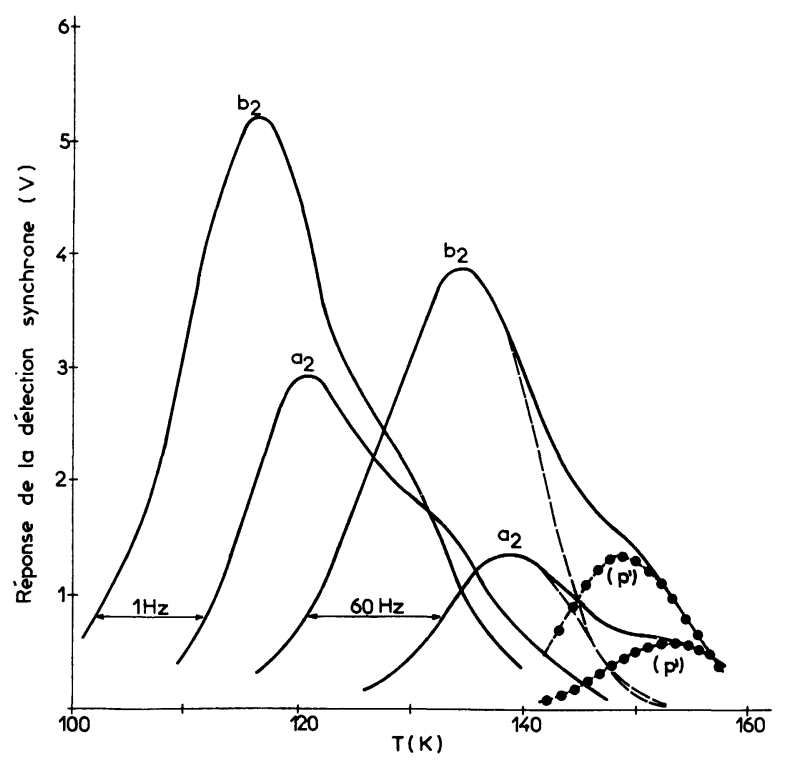

Fig. 6. - Partie basse température des spectres de $a_{2}$ et $b_{2}$ aux fréquences de 1 et $60 \mathrm{~Hz}$; les pics $\left(\mathrm{p}^{\prime}\right)$ et $(\mathrm{p})$ déduits de ces spectres sont tracés en pointillé pour $f=60 \mathrm{~Hz}$. Le substrat GaAs $\left(n=2 \times 10^{16} \mathrm{~cm}^{-3}\right)$ de la diode est multiimplanté en oxygène (dose : $2,2 \times 10^{11} \mathrm{~cm}^{-2}$ ).

[Low-temperature part of spectra $a_{2}$ and $b_{2}$ at frequencies 1 and $60 \mathrm{~Hz}$; the peaks $\left(\mathrm{p}^{\prime}\right)$ and $(\mathrm{p})$ deduced from these spectra are drawn in dotted lines for $f=60 \mathrm{~Hz}$. The GaAs substrate $\left(n=2 \times 10^{16} \mathrm{~cm}^{-3}\right)$ of the diode is oxygen multi-implanted (dose : $\left.2.2 \times 10^{11} \mathrm{~cm}^{-2}\right)$.] définitive c'est avec la mesure de $a_{2}$ qu'on obtient la meilleure sélectivité.

2.5 Evaluation de la SIGNATURE D'UN DÉFAUT. L'évaluation de la signature d'un défaut peut être menée à partir de l'une des expressions du tableau I qui dépendent chacune de deux inconnues : $x$ et $\Delta C_{0}$; mais en normalisant ces expressions à leur valeur maximale, elles ne sont plus fonction que de $x$. La résolution de l'une de ces équations normalisées détermine $x=\omega / e_{\mathrm{n}}$, c'est-à-dire en définitive la probabilité d'émission $e_{\mathrm{n}}$ en fonction de la température, un programme de régression linéaire permet d'évaluer $\sigma_{\infty}$ et la pente de la droite $\operatorname{Ln}\left(T^{2} / e_{n}\right)=f(1 / T)$ d'où on déduit l'énergie d'activation; on a donc ainsi la signature du piège en une seule remontée de température; d'autre part la variance $s$ de $E_{\mathrm{a}}$ permet d'estimer l'erreur sur cette grandeur et l'on peut montrer qu'une erreur sur $E_{\mathrm{a}}$ implique une erreur beaucoup plus importante sur $\sigma_{\infty}[8]$; pour une mesure plus précise de $\sigma_{\infty}$ il est préférable d'utiliser une autre méthode comme celle de Partin et al. [9]; l'erreur sur $E_{\mathrm{a}}$ est due surtout à l'erreur sur la température; dans les méthodes D.L.T.S. où l'on effectue plusieurs remontées de températures, on peut espérer des compensations statistiques des fluctuations aléatoires dans les mesures de températures et obtenir ainsi une précision sur $E_{\mathrm{a}}$ meilleure que celle obtenue avec une seule remontée de température.

Les méthodes ne retenant que le maximum du pic d'un spectre pour calculer la probabilité d'émission demandent, pour évaluer les mêmes caractéristiques, plusieurs spectres D.L.T.S. obtenus soit au moyen de plusieurs remontées de températures, soit en enregistrant plusieurs spectres en une seule remontée de température, mais il faut alors un appareillage plus complexe pour programmer cet enregistrement. Dans ces méthodes le nombre de valeurs de la probabilité d'émission $e_{\mathrm{n}}$ est limité par le nombre de spectres enregistrés, tandis que dans la méthode que nous proposons le nombre de valeurs de $e_{\mathrm{n}}$ déterminé est théoriquement aussi grand que l'on veut, ce qui permet de calculer l'énergie d'activation avec plus de précision lorsque le pic D.L.T.S. n'est pas perturbé par la présence d'un pic voisin; dans le cas contraire, le choix des points permettant d'évaluer $e_{\mathrm{n}}$ est limité à la partie non perturbée du pic.

3. Résultats expérimentaux. - La méthode décrite ici a été utilisée pour étudier des échantillons de GaAs, de type $n\left(n=2 \times 10^{16} \mathrm{~cm}^{-3}\right)$, implantés en oxygène. L'implantation d'oxygène a été faite pour obtenir une concentration en oxygène sensiblement constante de la surface à une profondeur de $2 \mu \mathrm{m}$. Après implantation, les échantillons sont encapsulés dans une couche de $1000 \AA$ de $\mathrm{Si}_{3} \mathrm{~N}_{4}$ puis recuits à $900^{\circ} \mathrm{C}$ pendant $15 \mathrm{~min}$. sous balayage d'argon; on enlève ensuite la couche de $\mathrm{Si}_{3} \mathrm{~N}_{4}$ par attaque chimique; enfin des contacts ohmiques et des contacts Schottky sont évaporés respectivement sur les faces arrière et avant. 
Les spectres D.L.T.S. obtenus pour ces échantillons sont composés de plusieurs pics; nous ne présentons (Fig. 6) que la partie basse température de quelques spectres pour montrer les particularités de la méthode que nous proposons; dans cette partie du spectre, un pic $(p)$ est superposé à un pic $\left(p^{\prime}\right)$ d'amplitude plus petite dont le maximum est à une température plus élevée que celle du pic (p). Aux températures plus élevées, il y a un autre pic (q) dans le spectre bien séparé de ses voisins, puis, au voisinage de la température ambiante, trois pics superposés dont on ne peut déduire les caractéristiques des défauts correspondants. La figure 6 montre que la séparation des deux pics (p) et $\left(\mathrm{p}^{\prime}\right)$ est meilleure à $60 \mathrm{~Hz}$ qu'à $1 \mathrm{~Hz}$ et aussi meilleure lorsqu'on enregistre $a_{2}$ que lorsqu'on enregistre $b_{2}$, conformément à ce que nous avons expliqué précédemment.

Pour comparer les résultats obtenus à partir des spectres des différents coefficients, nous présentons quelques courbes de $\log \left(T^{2} / e_{\mathrm{n}}\right)$ en fonction de $1 / T$ (déduites de l'amplitude $A_{2}$ aux fréquences de 1,5 , $60 \mathrm{~Hz}$ ) de $a_{1}$ aux fréquences 20 et $60 \mathrm{~Hz}$ (Fig. 7) et de $b_{2}, a_{2}, \phi_{2}$ à la fréquence de $60 \mathrm{~Hz}$ (Fig. 8). Nous constatons que pour le pic (q) bien séparé des autres

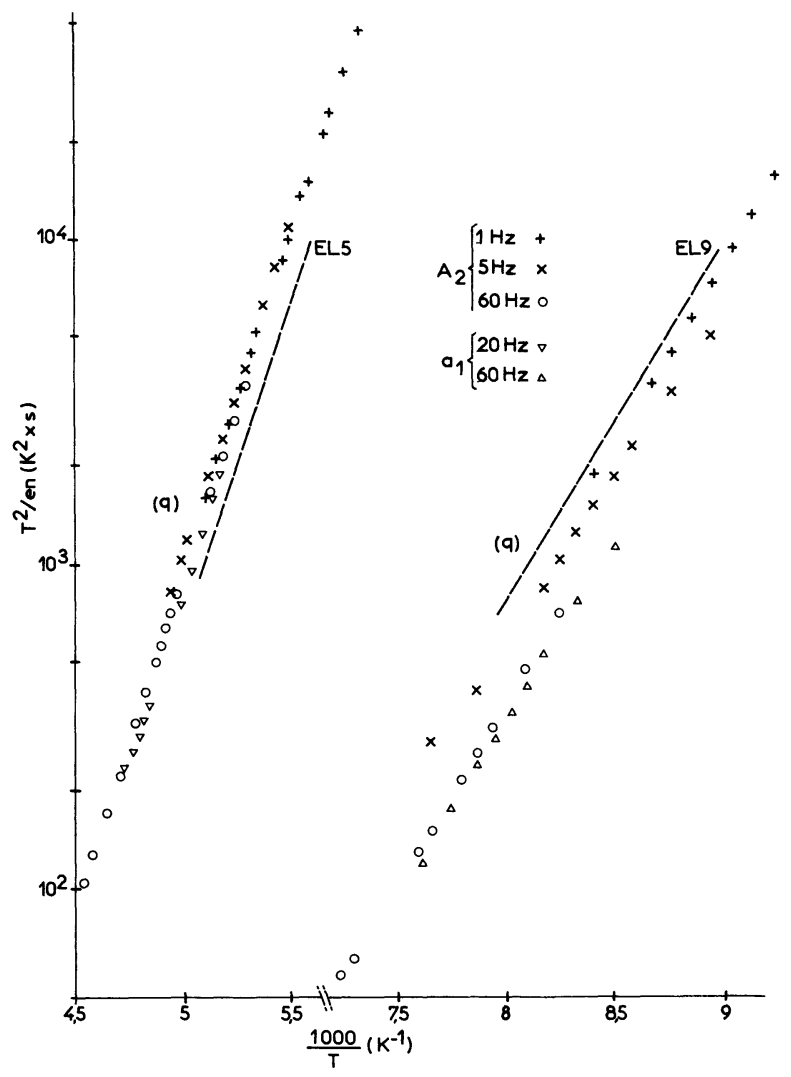

Fig. 7. - Variations de $T^{2} / e_{\mathrm{n}}$ en fonction de $1 / T$ déduites des spectres $A_{2}(f=1,5$ et $60 \mathrm{~Hz})$ et $a_{1}(f=20$ et $60 \mathrm{~Hz})$; EL5 et EL9 sont les niveaux profonds de la nomenclature de Martin et al. [6].

$\left[T^{2} / e_{\mathrm{n}}\right.$ variation as function of $1 / T$ deduced from spectra $A_{2}$ $(f=1.5$ and $60 \mathrm{~Hz})$ and $a_{1}(f=20$ and $60 \mathrm{~Hz})$; EL5 et EL9 are deep-states of Martin et al. [6] nomenclature.] pies dans le spectre, les valeurs de $\log \left(T^{2} / e_{\mathrm{n}}\right)$ coïncident aux incertitudes expérimentales près évaluées à quelques $\%\left(E_{\mathrm{a}}=0,39 \mathrm{eV}, s=0,005 \mathrm{eV}\right)$. Par contre, la perturbation du maximum du pic (p) par le pic $\left(p^{\prime}\right)$ augmente les incertitudes sur les valeurs de $e_{\mathrm{n}}$ déterminées par des mesures aux fréquences de $1,5,20$, $60 \mathrm{~Hz}$; on peut noter que les valeurs de $T^{2} / e_{\mathrm{n}}$ déterminées à partir des mesures de $a_{1}, a_{2}, b_{2}$ faites à la fréquence de $60 \mathrm{~Hz}$ (Figs. 7 et 8 ) coïncident mieux qu'aux autres fréquences, c'est en effet à cette fréquence que la séparation des pics (p) et ( $\mathrm{p}^{\prime}$ ) est la meilleure Pour l'état $(\mathrm{p}), E_{\mathrm{a}}$ déterminée à partir de $\phi_{2}$ a une valeur plus faible que celle déterminée à partir des autres coefficients : en effet la phase $\phi_{2}$ est évaluée à partir du rapport $b_{2} / a_{2}$; mais les deux pics $a_{2}$ et $b_{2}$ étant décalés en températures (Fig. 6) certaines valeurs de $\phi_{2}$ sont mesurées à partir du côté haute température du pic $b_{2}$ (perturbé par le pic $\left.\left(\mathrm{p}^{\prime}\right)\right)$ et du côté basse température du pic $a_{2}$ (non perturbé); ces dernières valeurs de $\phi_{2}$ ne sont donc pas précises et il en résulte une valeur plus petite de $E_{\mathrm{a}}$; l'erreur peut atteindre 15 à $20 \%$ suivant la fréquence de mesure (les deux pics étant plus ou moins superposés). La phase $\phi_{2}$ ne permet donc une détermination précise de la signature d'un état que lorsque le pic qu'il produit, est bien séparé des autres dans le spectre.

Nous présentons également pour le pic $\left(\mathrm{p}^{\prime}\right)$ les valeurs de $T^{2} / e_{\mathrm{n}}$ (Fig. 8) déduites des spectres de $a_{2}$ aux fréquences de 1 et $60 \mathrm{~Hz}$; pour cela on évalue $E_{\mathrm{a}}$ l'énergie d'activation du défaut correspondant au

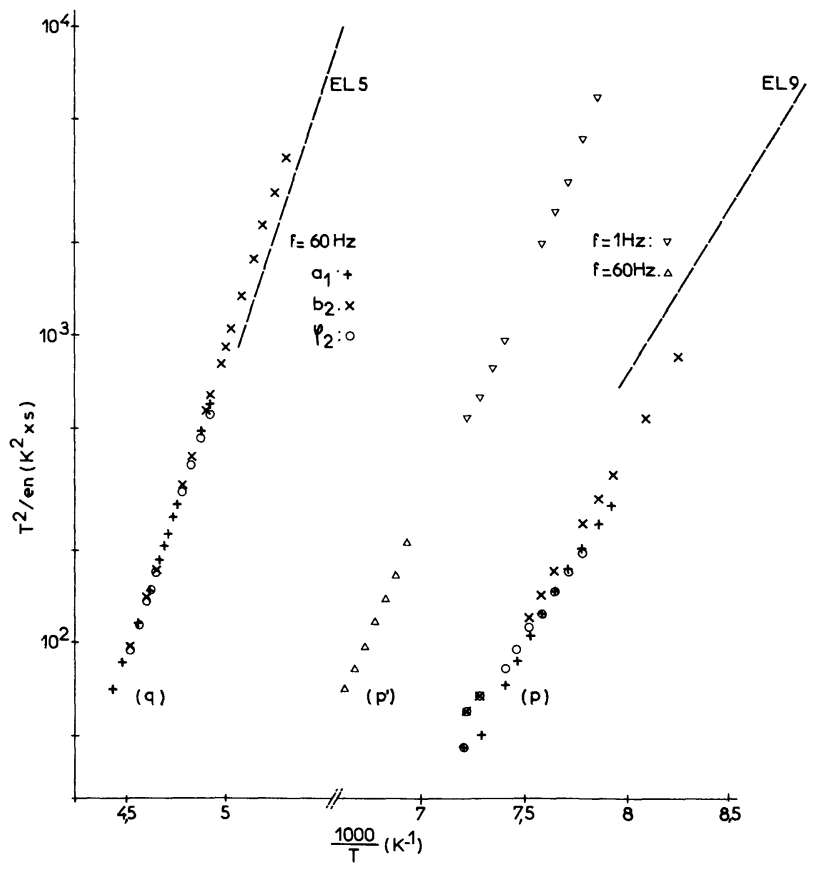

Fig. 8. - Variations de $T^{2} / e_{\mathrm{n}}$ en fonction de $1 / T$ déduites des spectres $a_{2}, b_{2}$ et $\phi_{2}(f=60 \mathrm{~Hz})$ pour les pics (p) et (q) et des spectres $a_{2}(f=1$ et $60 \mathrm{~Hz})$ pour le pic $\left(\mathrm{p}^{\prime}\right)$.

$\left[T^{2} / e_{\mathrm{n}}\right.$ variation as function of $1 / T$, deduced from spectra $a_{2}, b_{2}$ and $\phi_{2}(f=60 \mathrm{~Hz})$ for the peaks $(\mathrm{p})$ and $(\mathrm{q})$ and spectrum $a_{2}(f=1$ and $60 \mathrm{~Hz})$ for the peak $\left(\mathrm{p}^{\prime}\right)$.] 
pic (p) à partir de la partie non perturbée du spectre (côté basse température : figure 6); puis, pour un point de la partie perturbée (côté haute température du pic : figure 6), on calcule la valeur théorique du pic (p) qu'on déduit de la valeur expérimentale pour obtenir la valeur du pic $\left(\mathrm{p}^{\prime}\right)$ à ce point ; nous présentons à la figure 6 les pics $\left(\mathrm{p}^{\prime}\right)$ déduits des coefficients $a_{2}$ et $b_{2}$ à la fréquence de $60 \mathrm{~Hz}$; la déconvolution de ces deux pics n'a été possible que parce que le maximum du pic (p) n'est pratiquement pas modifié par le pic $\left(\mathrm{p}^{\prime}\right)$ d'amplitude nettement plus faible que celle du pic (p) (Fig. 6); l'énergie $E_{\mathrm{a}}$ calculée pour le pic $\left(\mathrm{p}^{\prime}\right)$ est $0,29 \mathrm{eV}(s=0,009 \mathrm{eV})$ : la précision sur $E_{\mathrm{a}}$ est donc moins bonne que sur les autres valeurs; on notera (Fig. 6) que la partie haute température du pic $\left(\mathrm{p}^{\prime}\right)$ coïncide avec la courbe expérimentale.

La signature de l'état $(\mathrm{p})$ est voisine de celle de l'état EL9 du catalogue de pièges à électrons de Martin et al. [6] ; l'énergie d'activation la plus précise $(0,22 \mathrm{eV}, s=0,006 \mathrm{eV})$ déterminée à $60 \mathrm{~Hz}$ est d'ailleurs la même que celle de cet état; le piège du catalogue précédent qui se rapproche le plus de l'état (q) $\left(E_{\mathrm{a}}=0,39 \mathrm{eV}\right)$ est $\operatorname{EL} 5(0,42 \mathrm{eV})$; nous n'avons trouvé aucun état du catalogue voisin de l'état $\left(p^{\prime}\right)$. Des mesures faites par la méthode de Lang sur les mêmes échantillons donnent pour les états $(p)$ et $(q)$ les mêmes résultats aux incertitudes expérimentales près [8].

4. Conclusion. - Dans cette méthode, l'échantillonneur-bloqueur réduit l'influence sur les mesures de la réponse transitoire du capacimètre et élimine la composante continue du signal de capacité (cela est aussi réalisé dans les méthodes où la fonction de pondération a une valeur moyenne nulle sur une période et dans la méthode de Lang); il limite aussi à une demi-période la durée d'application de ce signal à l'entrée de la détection synchrone, cette procédure permet d'obtenir des expressions simples pour les coefficients de Fourier $a_{1}, a_{2}, b_{2}$ et améliore leur rapport signal/bruit. Nous donnons les valeurs maximales de ces coefficients en fonction de la fréquence ainsi que les valeurs de $x=\omega \tau$ correspondantes; ces valeurs sont en effet nécessaires pour évaluer les probabilités d'émissions en une seule remontée de température. Nous montrons que la mesure du coefficient $a_{2}$ offre un meilleur pouvoir séparateur des pics du spectre que celui des autres coefficients. Nous caractérisons les défauts d'un échantillon de GaAs multiimplanté en oxygène pour illustrer la méthode. Le montage décrit peut être utilisé pour réaliser une méthode D.L.T.S. où la capacité de la diode est maintenue constante [10].

Remerciements. - Nous remercions G. Pelous du C.N.E.T. de Lannion et le Professeur Y. Colin de
l'Université de Rennes I pour l'aide et les conseils qu'ils nous ont apportés pour réaliser ce travail.

Appendice. - Le choix de $t_{\mathrm{d}}$ dépend de la constante de temps $\tau^{\prime}$ de la réponse du capacimètre que nous utilisons sur sa gamme de mesure la plus sensible ( $2 \mathrm{pF})$ en effectuant des mesures différentielles de capacité ; la variation brutale de la polarisation de la diode provoque une variation d'amplitude $A^{\prime} \mathrm{du}$ signal de sortie du capacimètre; lors du retour à l'équilibre (diode polarisée en inverse), l'expression du signal de sortie précédent en fonction du temps est donnée par la relation :

$$
A^{\prime} \exp \left(-t / \tau^{\prime}\right)
$$

La constante de temps $\tau^{\prime}$ a été évaluée par Gulberg [11] $:\left(\tau^{\prime}\right)^{-1}=3500 \mathrm{~s}^{-1}$. La réponse que donne la détection synchrone de ce signal est exprimée par l'une des relations du tableau I où l'on remplace $x$ par $x^{\prime}=\omega \tau^{\prime}$ et $\Delta C_{0}$ par $A^{\prime}$; dans le cas où $x^{\prime}$ est toujours petit devant 1 (à $\left.60 \mathrm{~Hz}, x^{\prime}=0,108\right)$, on peut donner des expressions approchées des réponses pour les différents coefficients de Fourier en négligeant $\exp \left(-\pi / x^{\prime}\right), x^{\prime 2}$ ou $4 x^{\prime 2}$ devant 1 ; dans le cas le plus défavorable $(f=60 \mathrm{~Hz})$ et pour $t_{\mathrm{d}}=1,6 \mathrm{~ms}$, les expressions des réponses s'écrivent alors :

$$
\begin{aligned}
& \text { - pour } a_{1}, a_{2}, A_{2}: \\
& s_{\mathrm{p}}=\frac{A^{\prime} x^{\prime}}{\pi} \exp \left(-t_{\mathrm{d}} / \tau^{\prime}\right)=1,27 \times 10^{-4} A^{\prime} \\
& \text { - pour } b_{2}: \\
& s_{\mathrm{p}}^{\prime}=\frac{2 A^{\prime} x^{\prime 2}}{\pi} \exp \left(-t_{\mathrm{d}} / \tau^{\prime}\right)=2,73 \times 10^{-5} A^{\prime}
\end{aligned}
$$

la réponse correspondant à $b_{2}$ (proportionnelle à $x^{\prime 2}$ ) est moindre que celle des autres coefficients.

Pour mieux apprécier la valeur de ces signaux parasites par rapport aux coefficients de Fourier, nous donnons, pour le coefficient $a_{2}, f=60 \mathrm{~Hz}$ et $\left(A^{\prime} / \Delta C\right)=10\left(\Delta C=0,2 \mathrm{pF}\right.$ car $A^{\prime} \simeq 2 \mathrm{pF}$ puisque la saturation de l'amplificateur du capacimètre limite cette valeur), la valeur du rapport du signal parasite $s_{\mathrm{p}}$ à la valeur maximale de $a\left(a_{2, \mathrm{~m}}\right)$ est $s_{\mathrm{p}} / a_{2 \mathrm{~m}}=0,038$; pour le coefficient $b_{2}, f=60 \mathrm{~Hz}$ et $\left(A^{\prime} / \Delta C\right)=10$, le rapport $\left(s_{\mathrm{p}} / b_{2, \mathrm{~m}}\right)=0,0031\left(b_{2, \mathrm{~m}}\right.$ valeur maximale de $b_{2}$ ).

Pour diminuer l'amplitude de ces signaux il faut diminuer soit la fréquence de mesure, soit $A^{\prime}$; on peut abaisser la valeur de $A^{\prime}$ en utilisant un interrupteur électronique qui isole la diode du capacimètre pendant l'impulsion de polarisation $[12,13]$. 


\section{Bibliographie}

[1] Lang, D. V., J. Appl. Phys. 45 (1974) 3023.

[2] Miller, G. L., Ramirez, J. V. and Robinson, D.A.H., J. Appl. Phys. 46 (1975) 2638.

[3] Schott, J. T., Deangelis, H. M. and White, W. R., Air Force Cambridge Laboratories Report $n^{\circ}$ A.F.C.R.L.-T.R. 760024 (1976).

[4] Day, D. S., Tsay, M. Y. and Streetman, B. G., J. Appl. Phys. 50 (1979) 5093.

[5] Pons, D., Thèse, Paris (1979).

[6] Martin, G., Mitonneau, A. and Mircea, A., Electron. Lett. 13 (1977) 191.

[7] Crowell, C. R., Alipanahi, S., Solid State Electron. 24 (1981) 25.
[8] Le Bloa, A., Favennec, P. N., Colin, Y., Phys. Status Solidi (a) 64 (1981) 85.

[9] Partin, D. L., Chen, J. W., Milnes, A. G. and Vassamillet, L. F., J. Appl. Phys. 50 (1979) 6845.

[10] Johnson, N. M., Bartelink, D. J., Schulz, M., The Physics of $\mathrm{SiO}_{2}$ and its interfaces, edited by S.T. Pantelides (Pergamon, New York) 1978, p. 421.

[11] Gulberg, J. Phys. E. 10 (1977) 1016.

[12] Balland, B., Marchand, J. J., Briot, R., Grange, G., J. Phys. E. 14 (1981) 367.

[13] Jansson, L., Kumar, V., Lebedo, L. A., Nideborn, K., J. Phys. E. 14 (1981) 464. 